Conclusion: To our knowledge, this is the first study demonstrating significantly lower scores among MDD relative non-MDD (based on PHQ-9) patients across all the 8 domains of LupusQoL; therefore, it adds to the current evidence that depression significantly impacts the quality of life of SLE. Predictors of MDD include older age, and involvement of the vascular, mucocutaneous, pulmonary, and musculoskeletal system. Additionally,a poor or fair description of self-rated health was a predictor of depression.

Disclosure of Interests: None declared

DOI: 10.1136/annrheumdis-2020-eular.946

\section{AB1225 LIPID PROFILE COMPARISON IN RHEUMATOID ARTHRITIS AND PSORIATIC ARTHRITIS: A CASE- CONTROL STUDY}

D. Á. Galarza-Delgado ${ }^{1}$, J. R. Azpiri-López ${ }^{2}$, I. J. Colunga-Pedraza ${ }^{1}$, A. Pérez Villar $^{1}$, I. C. Zárate Salinas ${ }^{2}$, P. F. Frausto Lerma ${ }^{1}$, M. A. Reyes Soto ${ }^{1}$, D. E. Flores Alvarado ${ }^{1}$, O. Ilizaliturri Guerra ${ }^{1}{ }^{1}$ Hospital Universitario Dr. José Eleuterio González, Rheumatology Department, Monterrey, Mexico; ${ }^{2}$ Hospital Universitario Dr. José Eleuterio González, Cardiology Department, Monterrey, Mexico

Background: Rheumatoid arthritis (RA) and psoriasic arthritis (PsA) are autoimmune diseases, in both diseases it has been described that the main cause of morbidity and mortality is cardiovascular (CV) disease. Dyslipidemia is the most recognized $\mathrm{CV}$ risk factor. An association is recognized between the concentrations of total cholesterol (TC), high-density lipoprotein cholesterol (HDL-C), low-density lipoprotein cholesterol (LDL-C), total triglycerides (TG), atherogenic index (AI) and the risk of myocardial infarction (MI), stroke and fatal cardiovascular disease (CVD). The relationship between serum lipid levels and CVD risk is potentially paradoxical in RA but this relationship has not been clarified in PsA. Objectives: To compare lipid profile between groups with RA, PsA and controls. Methods: A cross-sectional observational study was designed, which included 95 patients between 45-75 years who fulfilled the CASPAR classification criteria for PsA. 95 patients between 45-75 years who fulfilled the ACR / EULAR 2010 classification criteria for RA and 95 age-matched controls. Concentrations of CT, HDL-C, LDL-C, TG and atherogenic index were compared between the groups. Clinical measures were compared using one-way ANOVA or Kruskall-Wallis tests. Post-hoc analysis was performed with Bonferroni's correction. A $p \leq 0.05$ was considered statistically significant. The data was analyzed using the SPSS version 25 software package.

Results: In our study, no significant difference in LDL-C was found between RA and PsA, however post-hoc analysis was performed where we found higher LDL-C levels among RA patients compared with controls ( $p$ 0.025). RA patients had higher HDL-C than PsA patients ( $p$ 0.006) but PsA had a higher HDL-C than controls $(p$ 0.007). TC/HDL-C was higher in PsA than RA and controls $(p 0.050)$. PsA patients were the group with the lowest HDL-C levels $(p 0.007)$. In contrast RA were the groups with the highest HDL-C levels ( $p$ 0.007). (Table 1).

Table 1. Clinical parameters.

\begin{tabular}{lcccc}
\hline PARAMETER & RA & PSA & Controls & $p$ \\
\hline TC $^{\star}$ & $176.6 \pm 37.2$ & $176.3 \pm 35.9$ & $186.34 \pm 33.172$ & 0.089 \\
TG $^{* *}$ & $132.7(102.0-187.3)$ & $131.0(97.2-189.2)$ & $118.35(88.2-162.25)$ & 0.245 \\
HDL-C** & $50.7(42.1-62.6)$ & $46.7(37.4-53.9)$ & $51.7(41.3-60)$ & $\mathbf{0 . 0 0 7}$ \\
LDL-C $^{*}$ & $94.36 \pm 21.70$ & $97.71 \pm 30.12$ & $105.32 \pm 31.35$ & $\mathbf{0 . 0 2 5}$ \\
TC/HDL-C** $^{*}$ & $3.41(2.81-4.08)$ & $3.74(3.17-4.47)$ & $3.49(2.99-4.52)$ & $\mathbf{0 . 0 5 0}$ \\
\hline
\end{tabular}

*Data are reported in mean $\pm \mathrm{SD}$

${ }^{* *}$ Data is reported in median (IQR)

Conclusion: Patients with inflammatory joint diseases have more adverse lipid profiles than controls.

References:

[1] Pietrzak, A., Chabros, P., Grywalska, E., Kiciński, P., Pietrzak-Franciszkiewicz, K., Krasowska, D., \& Kandzierski, G. (2019). Serum lipid metabolism in psoriasis and psoriatic arthritis-an update. Archives of medical science: AMS, 15(2), 369

Disclosure of Interests: None declared

DOI: 10.1136/annrheumdis-2020-eular.5805

$\mathrm{AB} 1226$

\section{PREDICTING CARDIOVASCULAR EVENTS IN} PATIENTS WITH SPONDYLOARTHRITIS AND PSORIATIC ARTHRITIS: THREE RISK ALGORITHMS

A. S. Pinto ${ }^{1}$, F. Cunha Santos ${ }^{1}$, S. P. Dinis ${ }^{1}$, J. F. Ferreira ${ }^{1,2}$, C. Vaz ${ }^{1,2} .{ }^{1}$ Local Health Unit of Guarda, Guarda, Portugal; ${ }^{2}$ Beira Interior University, Covilhã, Portugal
Background: Inflammatory joint disorders have an increased cardiovascular (CV) risk when compared with general population. In 2009, the EULAR task force advocated the use of a 1.5 multiplication factor for these risk prediction models in Rheumatoid Arthritis patients with specific characteristics. The SCORE was not modified in patients with Psoriatic Arthritis (PsA) and Spondyloarthritis (SpA) in accordance with the present EULAR recommendations.

Objectives: To assess the accuracy of several CV risk algorithms to predict an event and determine its sensibility and specificity.

Methods: A retrospective analysis of PsA and SpA patients, followed in our department in Local Health Unit of Guarda during one year (2019) was done. We determined CV risk profile of our patients using the following data: gender, age, smoking status, blood pressure, lipid values and diabetes mellitus status. These variables were used to calculate risk prediction algorithms such as Framingham, the American College of Cardiology/American Heart Association (ACC/AHA) risk score and the Systematic Coronary Risk Evaluation (SCORE). Discriminatory ability for CV risk prediction was evaluated by the area under the ROC curves. Sensibility and specificity were calculated for low-to-intermediate and intermediate-to-high risk cut-offs. Cut-off values that mark the high risk were defined in $5 \%$ for SCORE, 20\% for Framingham and ACC/AHA.

Results: 112 caucasic patients were included, $61 \%$ female with a mean age of $52.15 \pm 14.18$ years and mean BMI of $27.11 \pm 4.81 \mathrm{~kg} / \mathrm{m}^{2} .7$ patients weren't eligible to apply these cardiovascular scores. 8 patients were identified with non-fatal cardiovascular events ( 2 cases of stroke, 5 cases of myocardial infarction and 1 case of thrombophlebitis). 69 patients diagnosed with $\mathrm{SpA}$ and 43 with PsA five CV events identified in SpA patients and three in PsA patients. Area unde the ROC in SpA were 0.729 (95\% Cl 0.461 to 0.996) for SCORE, 0.839 (95\% C 0.735 to 0.943 ) for Framingham and 0.804 (95\% Cl 0.683 to 0.925$)$ for ACC/AHA Area under the ROC in PsA patients were $0.603(95 \% \mathrm{Cl} 0.327$ to 0.879$)$ for SCORE, 0.660 (95\% Cl 0.326 to 0.995$)$ for Framingham and $0.804(95 \% \mathrm{Cl} 0683$ to 0.925$)$ for ACC/AHA. Sensibility and specificity were discriminated in table 1.

Table 1. Sensibility, specificity, positive predictive value (PPV) and negative predictive value (NPV) of CV risk algorithms

\begin{tabular}{lcccc}
\hline & Sensibility (\%) & Specificity (\%) & PPV (\%) & NPV (\%) \\
\hline SCORE > 1\% & & & & \\
SpA & 75 & 63 & 18 & 96 \\
PsA & 100 & 41 & 5 & 100 \\
SCORE > 5\% & & & & \\
SpA & NA & NA & NA & NA \\
PsA & 0 & 93 & 0 & 97 \\
Framingham >10\% & & & & \\
SpA & 83 & 78 & 28 & 98 \\
PsA & 50 & 51 & 5 & 95 \\
Framingham $>$ 20\% & & 86 & 20 & 93 \\
SpA & 33 & 69 & 8 & 96 \\
PsA & 50 & 77 & 29 & 94 \\
ACC/AHA $>$ 5\% & & 44 & 9 & 100 \\
SpA & 67 & & & \\
PsA & 100 & 86 & 14 & 88 \\
ACC/AHA $>\mathbf{2 0} \%$ & 17 & 83 & 14 & 97 \\
SpA & 50 & &
\end{tabular}

Conclusion: A good discrimination between patients with or without CV events has been demonstrated by area under the ROC curve, particularly to SpA patients. In PsA, the sample was smaller, which represents a limitation in this study. SCORE $>5 \%$ did not identify CV events, therefore sensibility couldn't be calculated. Overall, the algorithms studied presented a low sensibility, underestimating CV risk. This could be explained since disease-related factors are not reflected in these scores. In our opinion, better algorithms are needed to correctly assess cardiovascular risk algorithms for $\mathrm{SpA}$ and $\mathrm{PsA}$, since the majority of the events occur in patients with low-intermediate risk.

References:

[1] Agca R, Heslinga SC, Rollefstad S, et al. EULAR recommendations for cardiovascular disease risk management in patients with rheumatoid arthritis and other forms of inflammatory joint disorders: 2015/2016 update, Ann Rheum Dis 2017;76: 17-28.

Disclosure of Interests: None declared

DOI: 10.1136/annrheumdis-2020-eular.3972

\section{AB1227 PREVALENCE OF RHEUMATIC MUSCULOSKELETAL DISORDERS IN A RURAL AREA OF UPPER EGYPT: WHO-ILAR COPCORD BASED COMMUNITY STUDY}

S. Rashad ${ }^{1}$, E. Abda ${ }^{2}$, Z. Selim ${ }^{3}$, S. Hussein ${ }^{2}$, T. Metwally ${ }^{4}$, D. Fouad ${ }^{5}{ }^{1}$ Suez University, Rheumatology \& Rehabilitation, Suez, Egypt; ${ }^{2}$ Assiut University, Rheumatology \& Rehabilitation, Assiut, Egypt; ${ }^{3}$ Assiut, Rheumatology \& Rehabilitation, Assiut, Egypt; ${ }^{4}$ Suez University, Family Medicine, Suez, Egypt; ${ }^{5}$ South Egypt Cancer Institute, Radiology, Assiut, Egypt 
Background: Rheumatic musculoskeletal disorders (RMSDs) are a common cause of long term pain and physical disability. In developed countries, RMSDS are a major cause of absence from work and thus have a big financial burden on the country economic status. Several studies have been published the incidence and prevalence of RMSDs in different world countries and found to be widely variable. Estimation of the extend of the problem of RMSDs in developing world, especially in rural economies will help better understanding of the risk factors that contribute to the initiation and progression of these diseases and help the health care authorities to provide proper health program services in these areas to reduce the physical and financial burden of RMSDs (Bagher et al., 2011; Majumdar et al., 2015 and Usenbo, et al., 2015).

Objectives: To estimate the prevalence rate of RMSDs in a rural population in Upper Egypt.

Methods: A cross-sectional based study was carried out and included 3988 subjects of population (2013 females and 1975 males). Mean age of patients was (46.89 $\pm 15.25 y s)$. They proceeded 4 phases of World Health Organization/International League of Associations for Rheumatology community-oriented program for control of rheumatic diseases survey questionnaire WHO-ILAR Community Oriented Program for screening of rheumatic diseases. Modified Health Assessment Questionnaire (HAQ) was used to assess the disability severity. Individuals suspected to have any rheumatic diseases were subjected to full clinical examination, laboratory and radiological investigations to reach a final diagnosis. They were classified according to appropriate criteria of diagnosis of diseases.

Results: A prevalence rate of RMSDs was $16.22 \%$, more prevalence in females $(10.38 \%$ vs. $5.84 \%$ for males, $P=0.000)$. The mean age of patients with RRMSDSs were older $(46.89 \pm 15.25 \mathrm{yrs})$ than healthy individuals $(29.56 \pm 18.95$ yrs) $(P=0.0001)$ and with increasing age ( $\geq 45-\leq 55 \mathrm{yrs})$. The identified RMSDs were OA $(8.5 \%)$, Soft tissue rheumatism (STR) $(6.57 \%)$, spinal disorders (SD) $(6.47 \%)$, fibromyalgia $(\mathrm{FM})(0.60 \%)$, RA $(0.30 \%)$, arthralgia $(0.18 \%)$, SPAs $(0.15 \%)$, Gout(0.6\%), Pseudogout $(0.08 \%)$, SLE (0.5\%), JIA(0.03) and MCTD $(0.03 \%)$. The prevalence rates for the majority of RRMSDSs were higher in females and with increasing age. About two thirds of the patients had grade II disability.

Conclusion: The prevalence rate of RMSDs in a rural population $\geq 15 y e a r s$ in Upper Egypt has been estimated to be $16.22 \%$. The most prevalent RMSDs are OA, STR and SD causing the greatest burden of the disease. The predictive risk of RMSDs has to be assessed in future studies.

References:

[1] Majumdar A, Kumar SG, Nair D, Sujiv A. Musculoskeletal complaints and predictors of musculoskeletal pain among adults in rural puducherry. Indian J Palliat Care. 2015; 21(1):121-123.

[2] Usenbo A, Kramer V, Young T, Musekiwa A. Prevalence of Arthritis in Africa: A Systematic Review and Meta-Analysis. PLoS One. 2015; 10 (8):e0133858.

[3] Bagher OM, Golbarg M, Hossein S. Pattern of rheumatic diseases in two outpatient clinics in Iran: similarities with some different features. Indian J Med Sci. 2011; 65(1):7-17.

Disclosure of Interests: None declared

DOI: 10.1136/annrheumdis-2020-eular.779

\section{AB1228 COMPARATIVE ANALYSIS OF COMORBID PATHOLOGY IN PATIENTS WITH RHEUMATOID ARTHRITIS AND ANKYLOSING SPONDYLITIS}

T. Raskina ${ }^{1}$, O. Malyshenko ${ }^{1}$, E. Usova ${ }^{1}$, M. Letaeva ${ }^{1}$, J. Averkieva ${ }^{1}{ }^{1}$ Federal State Educational Institution of Higher Education "Kemerovo State Medical University," Kemerovo, Russian Federation

Background: Interest in comorbid conditions in rheumatoid arthritis (RA) and ankylosing spondylitis (AS) is due to their influence on the course and prognosis of the disease itself, the quality of life of patients and the choice of treatment tactics

Objectives: To compare the frequency and structure of comorbid pathology in patients with RA and AS.

Methods: 130 cases of patients with RA (95 women, 35 men, average age $47 \pm 6.6$ years) and 86 with AS ( 27 women and 59 men, average age $44 \pm 7.4$ years) were analyzed. The effect of concomitant pathology on the long-term prognosis was evaluated using the Charlson comorbidity index.

Results: Patients with RA had a combined pathology in $93.0 \%$ (122) cases, of which $24.5 \%$ (30) - one, $28.6 \%$ (35) - two, $46.7 \%$ (57) - three or more concomitant diseases. Concomitant diseases in AS patients were registered in $83.7 \%$ (72) of cases, including one in $36 \%$ (31), two in 14\% (12), and three or more in $33.7 \%(29)$. The Charlson index from 1 to 2 points was determined in $78.5 \%$ (102) of RA patients and in $77.9 \%(69)$ of patients with AS $(p=0.067)$. Values from 3 to 4 points had $19.2 \%(25)$ and $20.9 \%$ (18) of patients, respectively $(p=0.064)$. The Charlson index of more than 5 points was registered in $2.3 \%$ (3) patients with RA and $1.2 \%$ (1) patients with AS.

The leading place in the structure of comorbidity in the group of patients with RA was occupied by diseases of the digestive system, which was observed in $79.2 \%$ (103) of patients. In the second place in the same group were registered cardiovascular diseases (CVD) - in $48.5 \%$ (63) patients, the most common arterial hypertension
- in $36.9 \%$ (48). $27.6 \%$ (36) of patients had chronic pyelonephritis in combination with urolitiasis. Osteoporosis was detected in $20.0 \%$ (26) of RA patients.

In the group of patients with AS, diseases of the digestive system were also most frequently registered - in 76.7\% (66) of patients. CVD was observed in 41.9 $\%$ (36) patients, of which $39.5 \%$ (34) had hypertension. Kidney diseases were registered in $9.3 \%$ (8) of patients. Osteoporosis was detected in $32.5 \%$ (28) of patients with AS.

Comparative analysis of the structure and frequency of diseases of the digestive system in RA and AS revealed no significant differences. CVD was registered more often in RA than in AS (48.5\% vs $41.8 \%, p=0.037)$. There were significant differ ences in the frequency of hypertension in the study groups of patients: hypertension was observed more often in patients with as in $39.5 \%$ of cases, while in RA only in $36.9 \%$ ( $p=0.041$ ), which is probably due to long-term use of medium and high doses of NSAIDs as "basic therapy" for AS. Kidney diseases were more common in RA than in AS ( $27 \%$ vs $9.3 \%, p=0.022)$. Osteoporosis, in contrast, was most frequently detected in patients with AS than in RA patients $(32.2 \%$ vs $20 \%$, $\mathrm{p}=0.047)$.

Conclusion: Despite differences in the pathogenesis of RA and AS, the longterm prognosis, determined by both rheumatic disease and comorbidities, did not differ significantly. Concomitant pathology is an important component that determines the management of patients.

Disclosure of Interests: None declared

DOI: 10.1136/annrheumdis-2020-eular.5650

\section{AB1229 SAFETY OF BIOSIMILAR ETANERCEPT IN THE TREATMENT OF RHEUMATIC DISEASES: DATA FROM THE MEXICAN ADVERSE EVENTS REGISTRY (BIOBADAMEX)}

V. Rivera Teran ${ }^{1}$, M. Pérez Rodríguez ${ }^{2}$, D. Alpizar-Rodriguez ${ }^{3}$, F. IrazoquePalazuelos ${ }^{4}$, S. Carrilo 4 , S. Sicsik ${ }^{4}$, D. Vega-Morales ${ }^{4}$, D. Miranda ${ }^{4}$, A. Castillo ${ }^{4}$, J. C. Casasola ${ }^{4}$, C. F. Pacheco Tena ${ }^{4}$, J. F. Moctezuma ${ }^{4}$, F. Aceves ${ }^{4}$, A. Paz ${ }^{4}$,

S. Duran Barragan ${ }^{4}$, L. Barile ${ }^{4}$, N. Santana ${ }^{4}$, D. X. Xibille Friedmann ${ }^{4}$. ${ }^{1}$ Colegio Mexicano de Reumatología, Unidad de Investigación, Mexico City, Mexico; ${ }^{2}$ Instituto Mexicano del Seguro Social, Centro de Adiestramiento en Investigación Clínica, Mexico City, Mexico; ${ }^{1}$ Colegio Mexicano de Reumatología, Unidad de Investigación, Mexico City, Mexico; ${ }^{4}$ Colegio Mexicano de Reumatología, Mexico City, Mexico

Background: Access to biosimilar drugs in Mexico started on 2014. Although biosimilar drugs safety has proved comparability to originator drugs on trials information about its safety on real-life data is limited.

Objectives: To compare safety in terms of adverse events of biosimilar etanercept $(\mathrm{BEt})$ to originator etanercept (OEt) using information from the Mexican Adverse Events Registry (BIOBADAMEX).

Methods: BIOBADAMEX is a Mexican cohort that collects the information of biologic and biosimilar drugs used in patients with rheumatic diseases in public and private practice since 2016. Patients enrolled are followed- up yearly. For this study we included patients from 18 to 65 years old who were or are currently in treatment with OEt or BEt and analyzed the frequency of adverse events (AE), the severity and the outcome of these. Baseline time was considered at enrolment to the cohort. We used logistic regression to analyze univariable and multivariable associations. Results: At the time of analysis a total of 119 have received treatment with OEt, 38 with BEt. Mean follow up time was 1.35 years. Rheumatoid arthritis (RA) was the most common disease for all the groups followed by ankylosing spondylitis (AS) and psoriatic arthritis (PSA). Both groups had similar baseline characteristics (Table 1). AE occurred in $4(3.4 \%)$ patients with OEt and in $6(15.8 \%)$ with BEt (OR $0.2,95 \% \mathrm{Cl} 0.04-0.7)$. The most frequent $\mathrm{AE}$ in OEt group was allergic reaction, $(2(2.5 \%)$ of patients), and infections were the most frequent $A E$ in $B E$ group $(2(5.3 \%))$. Of patients with BEt, $2(5.3 \%)$ had severe AE compared to none in the OEt $(p=0.012)$. In the multivariable adjusted analysis comparing development of $A E$ vs no $A E$ including $B E t$, comorbidities and glucocorticoids, we found that use of $\mathrm{BEt}(\mathrm{OR} 4.6,95 \% \mathrm{Cl} 1.1-19.5)$ and presence of comorbidities (OR $4.6,95 \% \mathrm{Cl} 1.01-20.5)$, were associated with $\mathrm{AE}$. Use of glucocorticoids was not significantly associated.

Table 1. Baseline characteristics

\begin{tabular}{lccc}
\hline & $\begin{array}{c}\text { Originator etanercept } \\
(\mathbf{n}=119)\end{array}$ & $\begin{array}{c}\text { Biosimilar } \\
\text { Etanercept } \\
(\mathbf{n}=\mathbf{3 8})\end{array}$ & $\begin{array}{c}\text { Univariable } \\
\text { Analysis }\end{array}$ \\
OR(95\%Cl) \\
\hline Sex (female), $\mathrm{n}(\%)$ & $98(82.4)$ & $27(71.0)$ & $1.9(0.8-4.4)$ \\
Age, median (IQR) & $53.6(45-61)$ & $51.3(43-58)$ & $1.0(0.9-1.0)$ \\
Body Mass Index, median (IQR) & $27.5(23.4-32.5)$ & $26.7(24-29)$ & $1.0(0.9-1.1)$ \\
Diagnosis, $\mathrm{n}(\%):$ & $98(82.4)$ & $26(68)$ & 1 \\
$\quad$ Rheumatoid arthritis & $13(10.9)$ & $9(24.0)$ & $0.3(0.1-0.9)$ \\
Ankylosing spondylitis & $8(6.9)$ & $3(8.0)$ & $0.7(0.1-2.8)$ \\
$\quad$ Psoriatic arthritis & $41(34.5)$ & $14(40.0)$ & $0.3(0.3-1.5)$ \\
Comorbidities, $\mathrm{n}(\%):$ & & &
\end{tabular}

